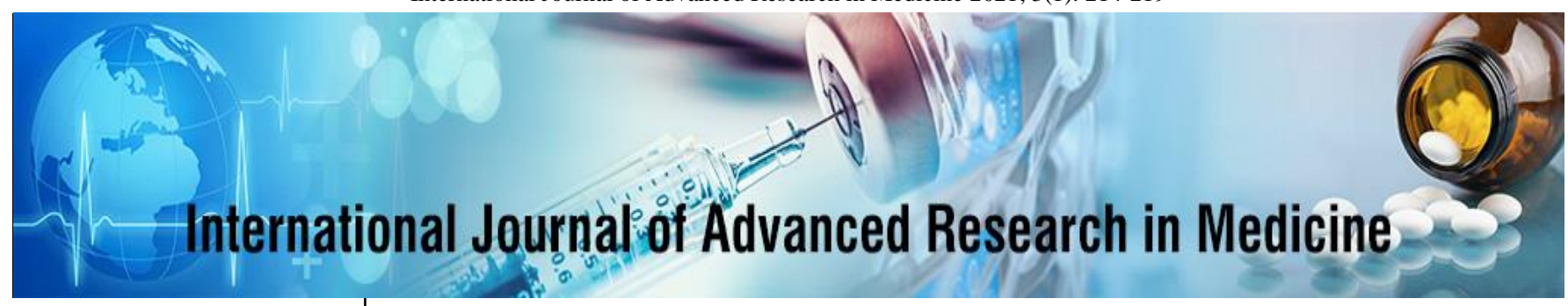

E-ISSN: 2706-9575

P-ISSN: 2706-9567

IJARM 2021; 3(1): 214-219

Received: 18-11-2020

Accepted: 22-01-2021

Dr. MS Ratna Lakshmi Deepika

Assistant Professor,

Department of General Medicine, NRI Medical College

\& Hospital, Chinakakani, Guntur, Andhra Pradesh, India
Corresponding Author: Dr. MS Ratna Lakshmi Deepika

Assistant Professor, Department of General Medicine, NRI Medical College \& Hospital, Chinakakani, Guntur, Andhra Pradesh, India

\section{A study on clinical profile and outcome of acute kidney injury of patients admitted in the intensive care unit of a tertiary care centre}

\author{
Dr. MS Ratna Lakshmi Deepika
}

DOI: https://doi.org/10.22271/27069567.2021.v3.i1d.141

\begin{abstract}
Background: Acute kidney injury is one of the most common conditions seen in the ICU setup. We come across acute kidney injury in patients on admission to the ICU initially, or as a development during the course of the disease, as a complication.

Aim \& Objectives: There is a need to study the most common diseases/ conditions causing AKI which can help in detecting the acute kidney injury at the earliest, and which will help in reducing the morbidity of patients.

Methodology: This is a prospective study of all the patients of AKI fitting into the inclusion criteria of the study who were admitted in the intensive care unit of NRI Medical College and Hospital, Guntur, a tertiary care centre, during the period of November 2019 to October 2020.

Results: In the present study, Incidence of AKI in the ICU was $6.7 \%$ with a male preponderance. Etiology of AKI was multifactorial. Incidence of medical related AKI was highest followed by medico surgical cases. Mortality was more in medical cases than medico surgical cases. Various comorbid factors play a role in final outcome of AKI. Type 2 diabetes mellitus was the highest predictor of mortality among the comorbid illnesses. Higher mortality was observed in the elderly age group. Most of the patients presented with hypotension and metabolic acidosis. In the setting of ICU despite of adequate treatment strategies including inotropes, dialytic modality and ventilator support, sepsis and multiorgan failure were the major causes of mortality in these patients. Among the risk, injury and failure groups, maximum number of patients from the failure group progressed to CKD and also had a terminal outcome. Mortality of patients of AKI in the ICU in our study was $8.14 \%$.

Conclusion: There is a need to avoid, or at the least, use the nephrotoxic drugs with caution in such patients. There is a constant relationship between the disease severity on the renal functions and the grade of AKI on the outcome of the disease. Hence there is a need to detect, evaluate and treat AKI at the earlier stages of the disease.
\end{abstract}

Keywords: chronic kidney disease, mortality, ICU, multifactorial incidence

\section{Introduction}

Acute kidney injury (AKI) is a heterogenous syndrome defined by a rapid (over hours to days) decline in the glomerular filtration rate (GFR) resulting in the retention of metabolic waste products, including urea and creatinine, and dysregulation of fluid, electrolyte, and acid- base homeostasis ${ }^{[1,2]}$.

Over the past decade, the term acute kidney injury has largely been replaced by the older term acute renal failure (ARF). The term acute renal failure suggested a dichotomous relationship between normal kidney function and overt organ failure; in contrast, the term acute kidney injury attempts to encompass the growing body of data associating small acute and transient decrements in kidney function with serious adverse outcomes ${ }^{[1]}$.

The kidney is among the few organs of the body with an ability to undergo virtually complete recovery of structure and function after being severely damaged. The potential for reversibility following an episode of transient ischemia or toxin induced cellular destruction underscores the need to provide patients with acute renal failure, irrespective of their cause, with every therapeutic option available.

It has been proved that kidney by itself is a source of variety of stem cells, simplest example of these cells helping repair, following injury is seen with acute kidney injury due to ischemic and toxic insult, where the renal tubular epithelial cells undergo regenerative response that leads to recovery of renal function ${ }^{[3]}$. 
Unfortunately, despite major advances in management of acute renal failure, the mortality in these patients is still high due to severe underlying illnesses and complications. It is rare for a patient to die directly as a result of renal failure. But it is an independent risk factor for death. Factors other than loss of renal function probably determine the outcome and their identification is necessary to improve the prognosis of acute kidney injury ${ }^{[4-7]}$.

AKI complicates $5 \%-7 \%$ of acute care hospital admissions and up to $30 \%$ of admission in ICU ${ }^{[1,2,8]}$. The present study was undertaken to evaluate the epidemiology, clinical features, mortality, morbidity and factors affecting outcome and prognosis of acute kidney injury. Understanding of these factors may help in early diagnosis and better treatment of ARF.

There is a need to study the most common diseases/ conditions causing AKI which can help in detecting the acute kidney injury at the earliest, and which will help in reducing the morbidity of patients.

\section{Need for the study}

Acute kidney injury is one of the most common conditions seen in the ICU setup.

We come across acute kidney injury in patients on admission to the ICU initially, or as a development during the course of the disease, as a complication.

Much of the available data on clinical course of patients with ARF is from western literature.

Compared to the western literature, reports from our country are scanty.

Also, the need for the present study in SDM is becoming increasingly important as the number of cases of acute kidney injury, especially in the ICU, is building up and the study regarding AKI in tertiary care centre.

\section{Aims and objectives of the study}

1. To analyse the causes, clinical profile of acute kidney injury in the ICU.

2. To analyse the risk and prognostic factors of acute kidney injury in the ICU.

3. To study the impact of acute kidney injury on the disease and final outcome of kidney function in the patients with acute kidney injury in the ICU.

\section{Source of data}

This is a prospective study of all the patients of AKI fitting into the inclusion criteria of the study who were admitted in the intensive care unit of NRI Medical College and Hospital, Guntur, a tertiary care centre, during the period of November 2019 to October 2020.

Totally, 1285 patients were admitted to the ICU during the study period. A total number of 86 cases were taken into the study.

Obstetrics and Gynecology were not taken as they were not fitting into the inclusion criteria

\section{Methods of collection of data}

Consecutive patients who came into the inclusion criteria in the study period were enrolled in study. They were assessed, investigated, and treated as per the existing practices without disturbing their routine protocol. Institute's Ethics committee approval was obtained for this study. The enrolled patient's demographic data including sex, address, occupation, marital status were noted. Patient's premorbid illness was noted including history of alcohol abuse and smoking. Patient's history and detail examination was done.

\section{Investigations}

The following investigations were done (as and when required)

- Complete urine examination, 24 hour urine protein estimation, urine albumin.

- Biochemical tests including

- Random blood glucose,

- Blood urea,

- Serum creatinine,

- Serum electrolytes,

- Serum total and differential bilirubin,

- Serum total protein, albumin, globulin, calcium, phosphorus, uric acid

- Alkaline phosphatase and transaminases.

- Complete hematological tests including coagulation profile,

- Bacterial and fungal (in patients with indication) cultures of blood, urine, venous catheters, and endotracheal secretions.

- Radiological tests include X-ray of chest and abdomen and ultrasonography/computer tomography (CT) of abdomen and pelvis.

- HIV, HBs Ag, HCV

- Ophthalmology- Fundoscopy.

- Renal biopsy

- $\mathrm{ABG}$

\section{Inclusion Criteria}

All patients admitted to ICU with wide variety of illness who develop AKI.

1. Patients who fulfill the RIFLE criteria for acute kidney disease (Risk, injury, failure). Risk- Creatinine x 1.5/ $<0.5 \mathrm{ml} / \mathrm{kg} / \mathrm{hr} \quad \mathrm{x}$ 6hrs. Injury- Creatinine $\mathrm{x} \quad 2 \quad /$ $<0.5 \mathrm{ml} / \mathrm{kg} / \mathrm{hr}$ x $12 \mathrm{hrs}$ Failure- Creatinine $\mathrm{x} 3$ or Creatinine $\geq 4 \mathrm{mg} / \mathrm{dl}$ or acute rise $>0.5 \mathrm{mg} / \mathrm{dl}$. OR < $0.3 \mathrm{ml} / \mathrm{kg} / \mathrm{hr} \times 24$ hours, or anuria x 12 hours

2. Minimum of 24 hours of admission

\section{Exclusion Criteria}

1. Patients of established Chronic Kidney Disease and end stage renal disease.

2. Prerenal factors like volume depletion which is correctable within 48 hours.

3. Age $<18$ years.

4. Discharge against medical advice.

5. Deaths within one day of admission.

Table 1: Sex distribution

\begin{tabular}{|c|c|c|}
\hline & No. Of patients & Percentage of total \\
\hline Males & 59 & 68.6 \\
\hline Females & 27 & 38.4 \\
\hline Total & 86 & 100 \\
\hline
\end{tabular}

Out of 86 patients included in the study, $59(68.6 \%)$ were males and $27(31.4 \%)$ were females 


\section{Age incidence and mortality}

Table 2: Age incidence and mortality

\begin{tabular}{|c|c|c|c|c|}
\hline Age (years) & No of patients & \% of total & Mortality in each Age group & \% mortality Of total \\
\hline$<20$ & 4 & 4.6 & 1 & 14.25 \\
\hline $21-30$ & 12 & 14 & 0 & 0 \\
\hline $31-40$ & 16 & 18.6 & 1 & 14.25 \\
\hline $41-50$ & 13 & 15 & 1 & 14.25 \\
\hline $51-60$ & 15 & 17.4 & 1 & 14.25 \\
\hline$>60$ & 26 & 30.4 & 3 & 43 \\
\hline Total & 86 & 100 & 7 & 100 \\
\hline
\end{tabular}

This study excludes patients $<18$ years.

In adult population, majority of the cases were in the age group of $>60$ years, the mortality in this age group was $11.53 \%$ which was $43 \%$ of total mortality.

There were 4 patients in the age group $<20$ years, with a mortality of $14.25 \%$
There were 12 patients in the age group of 21-30 years with no mortality in the age group.

There were 16, 13 and 15 patients in the age group of 31-40, $41-50,51-60$ with a mortality of $14.25 \%$ in each group Thus, higher mortality was found in patients $>60$ years.

Table 3: Etiology

\begin{tabular}{|c|c|c|c|c|}
\hline Etiology & No. of patients & \% & Mortality & \% mortality \\
\hline Medical & 81 & 94.18 & 6 & 74.04 \\
\hline Malaria & 6 & 7.4 & 0 & \\
\hline Vhf & 19 & 23.5 & 4 & \\
\hline Pneumonia & 9 & 11.1 & 1 & \\
\hline Acute ge & 33 & 40.7 & 1 & \\
\hline Uti & 12 & 14.8 & 0 & \\
\hline Snake bite & 1 & 1.3 & 0 & \\
\hline Rickettsial Disease & 1 & 1.3 & 0 & 20 \\
\hline Medicosurgical & 5 & 5.82 & 1 & \\
\hline Snake bite with Cellulitis & 2 & 40 & 0 & \\
\hline Pancreatitis & 1 & 20 & 0 & \\
\hline Obstructive Uropathy & 1 & 20 & 0 & \\
\hline Perforation Peritonitis & 1 & 20 & 1 & 7 \\
\hline Total & 86 & & & \\
\hline
\end{tabular}

In this study, there were $81(74.04 \%)$ cases due to medical causes. These include 6 cases of malaria, 19 cases of viral haemorrhagic fever, 9 cases of pneumonia, 33 cases of acute gastroenteritis, 12 cases of urinary tract infection, 1 case of snake bite and cases of 1 rickettsial disease.

Out of the 6 malaria patients, 2 were Plasmodium falciparum, 1 patient of Plasmodium vivax and 3 patients were of mixed falciparum and vivax species.

5 patients $(20 \%)$ were medicosurgical. 2 cases of snake bite with cellulitis, 1 each of pancreatitis, perforation peritonitis and obstructive uropathy.

\section{Mortality}

The overall mortality was $8.14 \%$.

The mortality in the medical group was $6.68 \%$ and was the highest; mortality in the surgical group was $1.46 \%$.

\section{Comorbid Conditions}

Table 4: Comorbid conditions

\begin{tabular}{|c|c|c|c|c|}
\hline Comorbidity & $\begin{array}{c}\text { No. Of } \\
\text { Patients }\end{array}$ & $\begin{array}{c}\% \text { of } \\
\text { Total }\end{array}$ & Mortality & $\begin{array}{c}\text { Percentage } \\
\text { Mortality }\end{array}$ \\
\hline Type 2 diabetes Mellitus & 15 & 17.44 & 1 & 14.28 \\
\hline Hypertension & 12 & 13.95 & 0 & 0 \\
\hline Cad & 5 & 5.81 & 0 & 0 \\
\hline
\end{tabular}

Out of 86 patients studied, 15(17.44\%) patients had T2DM, $12(13.95 \%)$ were hypertensives, $5(5.81 \%)$ had coronary artery disease.
Higher mortality was seen among the diabetic group which was $14.28 \%$.

\section{Presenting features}

Table 5: Presenting features

\begin{tabular}{|c|c|c|}
\hline Presenting features & No. of patients & \% of total patients \\
\hline Urinary complaints & 9 & 11.5 \\
Anuria & 11 & 12.8 \\
\hline Oliguria & 7 & 8.5 \\
\hline Fluid overload & 22 & 26 \\
\hline Hypotension & 14 & 16.8 \\
\hline Encephalopathy & 21 & 25.4 \\
\hline Metabolic acidosis &
\end{tabular}

Total anuria was present in $9(11.5 \%)$ patients out of which 3 cases were of acute gastroenteritis, 2 of viral haemorrhagic fever, 1 of UTI with sepsis, 1 of pneumonia with sepsis, 1 of obstructive uropathy and 1 of perforation peritonitis Oliguria was present in $11(12.8 \%)$ patients.

Rest of the patients had non-oliguric renal failure. Fluid overload was present in 7 patients, all of them presenting with pulmonary edema. Hypotension was observed in 22 patients- 13 patients were of acute gastroenteritis, 3 were of pneumonia, 3 of UTI, 1 of viral haemorrhagic fever, malaria and perforation peritonitis each. Encephalopathy was seen in $14(16.8 \%)$ patients. Metabolic acidosis was seen in 21 $(25.4 \%)$ of patients. 


\section{Type of renal failure and mortality}

Table 6: Type of renal failure and mortality

\begin{tabular}{|c|c|c|c|c|}
\hline $\begin{array}{c}\text { Type of renal } \\
\text { failure }\end{array}$ & $\begin{array}{c}\text { No. of } \\
\text { patients }\end{array}$ & Incidence & Mortality & $\begin{array}{c}\text { Percent } \\
\text { mortality }\end{array}$ \\
\hline Prerenal & 25 & 29.1 & 1 & 4 \\
\hline Intrinsic renal & 60 & 69.75 & 6 & 10 \\
\hline Post renal & 1 & 1.15 & 0 & 0 \\
\hline
\end{tabular}

Prerenal ARF was seen in 26 patients, hence incidence was $29.1 \%$. Mortality was $4 \%$ in this group.

Intrinsic renal failure was seen in 58 patients, hence incidence was $69.75 \%$.

Mortality was $10 \%$ in this group.

Post-renal ARF was seen in 1 patient with an incidence of $1.15 \%$ and this group had no mortality.

Hence least mortality was observed in postrenal ARF but it was statistically not significant.

\section{Complications}

Table 7a: Complications

\begin{tabular}{|c|c|c|c|c|}
\hline Complications & $\begin{array}{c}\text { No. of } \\
\text { patients }\end{array}$ & $\begin{array}{c}\text { Percent of } \\
\text { total }\end{array}$ & Mortality & $\begin{array}{c}\text { Percent } \\
\text { Mortality }\end{array}$ \\
\hline MODS & 36 & 41.86 & 6 & 16.67 \\
\hline GI Haemorrhage & 3 & 3.49 & 0 & 0 \\
\hline Sepsis & 40 & 46.51 & 3 & 7.5 \\
\hline
\end{tabular}

MODS- Multiorgan Dysfunction Syndrome- defined as dysfunction of more than 1 organ within 24 hours requiring intervention to maintain homeostasis.

MODS was seen in a total of 36 patients $(41.86 \%)$ out of which 6 expired (16.67\%). 5 patients of Complicated malaria, 20 patients of viral haemorrhagic fever, 7 patients of pneumonia, 2 patients of UTI, 1 patient each of snake bite and perforation peritonitis developed MODS.

Table 7b: No. of organs involved in MODS

\begin{tabular}{|c|c|c|c|}
\hline & $\mathbf{4}$ & $\mathbf{3}$ & $\mathbf{2}$ \\
\hline Complicated malaria & 0 & 5 & 0 \\
\hline VHF & 2 & 13 & 5 \\
\hline Pneumonia & 3 & 3 & 1 \\
\hline UTI & 0 & 1 & 1 \\
\hline Snake bite & 0 & 0 & 1 \\
\hline Perforation peritonitis & 0 & 1 & 0 \\
\hline
\end{tabular}

GI haemorrhage was seen in the form of malena in $3(3.49 \%)$ patients.
Sepsis was seen in $40(46.51 \%)$ patients out of which 3 $(7.5 \%)$ expired.

Blood culture was positive in 5 patients- 4 being ESBL $E$. coli, 1 being Acinetobacter sps.

Urine culture was positive in 9 patients,- 7 being ESBL E.coli, 1 being ESBL Pseudomonas species, 1- ESBL Klebsiella 52neumonia.

Out of these, 2 patients isolated ESBL E.coli from both urine and blood. ET culture was positive in 6 patients- with one each of ESBL Klebsiella pneumonia, Pseudomonas, Acinetobacter sps, MRSA, Streptococcus and Mycobacterium tuberculosis being isolated.

Pus culture was positive for 2 patients of snake bite with cellulitis- 1 being MRCONS and the other being Streptococcus.

\section{Ventilation and mortality}

Table 8: Ventilation and mortality

\begin{tabular}{|c|c|c|c|}
\hline No. Of patients & \% of total & Mortality & \% Mortality \\
\hline 10 & 11.6 & 6 & 60 \\
\hline
\end{tabular}

Out of 19 cases of VHF with MODS and 9 patients of Pneumonia, $10(11.6 \%)$ patients were intubated and $6(60 \%)$ patients expired.

ET culture was positive in 6 patients- with one each of ESBL Klebsiella pneumonia, Pseudomonas, Acinetobacter sps, MRSA, Streptococcus and Mycobacterium tuberculosis being isolated.

\section{Mode of treatment}

Table 9a: Mode of treatment

\begin{tabular}{|c|c|c|c|c|}
\hline & Risk & Injury & Failure & Total \\
\hline Conservative Management & 23 & 27 & 18 & 68 \\
\hline Haemodialysis & 0 & 1 & 17 & 18 \\
\hline
\end{tabular}

Out of 86 patients, $68(79 \%)$ patients were managed conservatively and $18(21 \%)$ needed haemodialysis. Out of the patients treated conservatively, 2(28.57\%) patients expired.

Table 9b: No. of cycles of HD required

\begin{tabular}{|l|l|l|l|}
\hline & $\geq \mathbf{3}$ & $\mathbf{2}$ & $\mathbf{1}$ \\
\hline No. of patients & 15 & 2 & 1 \\
\hline & & & \\
\hline
\end{tabular}

Outcome

Table 10: Outcome

\begin{tabular}{|c|c|c|c|c|c|c|c|}
\hline & Total & $\begin{array}{c}\text { Complete recovery } \\
\text { (no. Of Patients) }\end{array}$ & \% of total & $\begin{array}{c}\text { Progressi on to ckd } \\
\text { (no. Of Patients) }\end{array}$ & \% of total & $\begin{array}{c}\text { Expired (no. of } \\
\text { patient S) }\end{array}$ & \% of tot al \\
\hline Risk & 23 & 22 & 93 & 0 & 0 & 1 & 4 \\
\hline Injury & 28 & 26 & 96 & 1 & 4 & 1 & 4 \\
\hline Failur E & 35 & 25 & 71 & 5 & 14 & 5 & 14 \\
\hline
\end{tabular}

Out of the total of 86 patients enrolled, 73 (84.88\%) had complete recovery, $6(6.9 \%)$ progressed to CKD and $7(8.13 \%)$ patients expired.

In our study, the total number of patients in the Risk, Injury and Failure groups were- 23 (26.74\%), 28 (32.55\%) and 35 $(40.7 \%)$ patients respectively. Out of these, $22(96 \%), 26$
(93\%) and $25(71 \%)$ of patients of the groups Risk, Injury and Failure had complete recovery. 1 patient each of risk and injury (4\% each) and 5 patients (14\%) of failure groups expired. Progression to CKD was seen in $1(4 \%)$ and 5 $(14 \%)$ patients each of Injury and failure groups. 


\section{Discussion}

The patient study includes acute kidney injury patients admitted to the ICU at NRI Medical College \& Hospital, Guntur.

The study comprised of 86 patients out of 1285 admitted to the ICU. Out of 86 patients, 59 were males and 27 were females. All patients were evaluated by appropriate history, clinical examination and laboratory investigations proforma. Incidence of AKI in our study was $6.7 \%$

The reported incidence of AKI in the ICU has varied widely ranging between 4 to $35.8 \%{ }^{[8]}$. This marked variability may be due to differences in population involved in the study, type of tertiary care facility available and basic underlying disease causing AKI. The reason for relatively lower incidence of AKI in our study may be due to the fact that majority of our patients developed AKI complicating various treatable forms of AKI in the ICU.

Similarly, in the study of Acute Renal Failure in intensive care unit by J Prakash, A.S. Murthy, incidence was $3.79 \%$ ${ }^{[8]}$. In a similar study conducted in Riyadh Intensive care by Marlies Ostermann, Rene. W.S. Chang, the incidence of AKI in ICU was $35.8 \%{ }^{[9]}$.

In our study, sex ratio was skewed more towards males being- $68.6 \%$. However in the study conducted by J Prakash et al. ${ }^{[8]}$, incidence of AKI in males was $56.5 \%$. In the study conducted by Marlies Ostermann in Riyadh ${ }^{[9]}$, 65\% were males. In our study, higher mortality was found in older age group of $>60$ years $(43 \%)$.

According to $\mathrm{J}$ Prakash et al. ${ }^{[8]}$, the mean age of patients getting admitted to the ICU was 44.9 years and there was no statistical significance between survivors and non survivors which was similar to our study.

Commonly noted co morbidities in our patients were Type 2 diabetes mellitus, hypertension and coronary artery disease of which diabetes was predominant (14.38\%). However the association was not statistically significant with regard to outcome.

Mehta RL et al. ${ }^{[10]}$ in the PICARD experience, have reported an incidence of comorbidity in excess of $30 \%$.

$\mathrm{J}$ Prakash et al. noted comorbidity in $52.17 \%{ }^{[8]}$. In our study, AKI due to medical causes was contributory in $94.18 \%$. The remaining $5.82 \%$ was contributed by surgical causes. The obstetric causes were excluded from our study as they did not meet the inclusion criteria.

J Prakash et al. ${ }^{[8]}$ showed results of $71.7 \%$ due to medical causes, $23.9 \%$ due to surgical cause and $4.3 \%$ due to obstetrical causes.

According to Cenzig et al. ${ }^{[11]} 59 \%$ had medical causes, $25 \%$ had surgical cause and $16 \%$ had obstetric cause contributing to ARF.

The overall mortality in our study was $8.14 \%$ of which mortality in the medical group was $6.68 \%$ and the remaining $1.46 \%$ was contributed by medico-surgical group.

According to the study done by Kennedy et al. ${ }^{[12]}$, mortality in medical group was $24 \%$, medico-surgical group was $28.5 \%$ and in obstetric was $1 \%$.

In our study hypotension was the predominant presenting features which was seen in $26 \%$ of the patients followed by metabolic acidosis- $25.4 \%$, encephalopathy was seen in $16.8 \%$, oliguria in $12.8 \%$ and fluid overload was seen in $8.5 \%$.

J Prakash et al. ${ }^{[8]}$ noted hypotension, altered sensorium and respiratory distress as the commonest features in the ICU.
$65 \%$ of patients were oliguric and $36.9 \%$ of patients had evidence of fluid overload.

Similarly, Mehta et al. ${ }^{[11]}$, in Spectrum of ARF in ICU: The PICARD experience, 2004 have noted hypotension in $20 \%$ of patients as the presenting feature which is comparable to that in our study.

Incidence of prerenal failure was less in our study as most of the patients who presented to us had already progressed to acute tubular necrosis. The percentage of prerenal, intrinsic renal and post renal AKI was $29.1 \%, 69.75 \%$ and $1.15 \%$ respectively and mortality in the prerenal and intrinsic renal groups being $4 \%$ and $10 \%$ with no mortality in the postrenal AKI group.

According to Mehta et al. ${ }^{[11]}$, prerenal ARF was seen in $50 \%$ of patients, $48 \%$ had intrinsic renal failure and $2 \%$ had postrenal ARF. Kaufman et al. ${ }^{[13]}$, showed a mortality of only $7 \%$ in prerenal AKI. The group with intrinsic renal failure had the highest mortality of $55 \%$.

In our study sepsis (46.51\%), MODS (41.86\%) and GI haemorrhage $(3.49 \%)$ were the main complications. Highest mortality was found in patients with MODS (16.67\%). Patients with sepsis had a mortality of $7.5 \%$. J Prakash et al. ${ }^{[8]}$, reported evidence of sepsis in $69.2 \%$ patients and mortality of $84 \%$. MODS was noted in $63 \%$ of cases and $83 \%$ of those had mortality.

In the study done by Marlies Ostermann et al. ${ }^{[9]}$, the incidence of MODS of patients in ICU was $23.1 \%$ with an overall mortality of $78.85 \%$. In our study $11.6 \%$ of the patients needed mechanical ventilation and the mortality in ventilated patients was $60 \%$. In the study by J Prakash et al. ${ }^{[8]}, 31$ patients $(67.4 \%)$ required ventilation out of which 23 patients $(74.21 \%)$ expired. According to Marlies Ostermann et al. ${ }^{[9]}, 57.5 \%$ patients needed mechanical ventilation of which $80.8 \%$ had terminal outcome.

In our study, out of 86 patients, $68(79 \%)$ had conservative line of management and 18 (21\%) underwent haemodialysis, out of which 2 patients $(28.5 \%)$ and 5 patients $(71.5 \%)$ expired in each group respectively.

J Prakash et al. ${ }^{[8]}$ showed mortality of $80 \%$ in dialysed group and $42 \%$ in non- dialysed group.

Metcalfe et al. ${ }^{[14]}$ in his study showed $73.5 \%$ of patients receiving RRT for ARF expired. This seems to be due to confounding effects of severity of underlying disease process, sepsis and MODS.

In our study, the total number of patients in the Risk, Injury and Failure groups were- $23(26.74 \%), 28(32.55 \%)$ and 35 $(40.7 \%)$ patients respectively. Out of these, 22 (96\%), 26 $(93 \%)$ and $25(71 \%)$ of patients of the groups Risk, Injury and Failure had complete recovery. 1 patient each of risk and injury (4\% each) and 5 patients $(14 \%)$ of failure groups expired. Progression to CKD was seen in $1(4 \%)$ and 5 (14\%) patients each of Injury and failure groups.

According to the study done by Marlies Ostermann et al. ${ }^{[9]}$, $17 \%$ patients were in the risk group, $11 \%$ had injury and $7.6 \%$ had failure. Mortality in respective groups were $20.9 \%, 45.6 \%$ and $56.8 \%$ respectively.

\section{Limitations of the study}

1. Obstetrical cases were not taken into the study as they were not fitting into the inclusion study.

2. Investigations like urinary sodium, FeNa, urinary osmolality are not available in our hospital and hence not studied. 
3. Only Haemodialysis and Peritoneal dialysis are available in our hospital and hence were used as modalities of RRT. CAVHD and CVVHD are not available in our institute.

\section{Conclusion}

From the studies done on AKI, we can infer that AKI is seen mostly in the elderly patients in the ICU and the outcome of the renal function is poorer when associated with comorbid conditions. Also the severity of illness, associated sepsis and MODS are the other prognostic factors. We can see the trend of increasing mortality among the three grades with the severity of AKI increasing from risk to failure. The RIFLE criteria was found to be a good predictor of recovery of renal function. Thus there is a need to monitor the renal functions in the form of urine output and biochemical parameters regularly in the patients in the ICU as we do with the other organs as in regular monitoring of cardiac status, CNS etc. There is a need to avoid, or at the least, use the nephrotoxic drugs with caution in such patients. There is a constant relationship between the disease severity on the renal functions and the grade of AKI on the outcome of the disease. Hence there is a need to detect, evaluate and treat $\mathrm{AKI}$ at the earlier stages of the disease.

\section{Acknowledgment}

The author is thankful to Department of General Medicine for providing all the facilities to carry out this work.

\section{References}

1. Asif Sharfuddin A, Steven Weisbord D, Paul Palversky M, Bruce Molitoris A. Acute Kidney Injury: Brenner BM. Brenner and Rector's- The Kidney, 9 ${ }^{\text {th }}$ ed. Philadelphia: Saunders- Elsevier 2012, 1044-1099.

2. Sushrut Waikar S, Joseph Bonventre V. Acute Kidney Injury: Fauci, Braunwald, Kasper, editors. Harrison's principles of internal medicine, $18^{\text {th }}$ ed, New York: Mc Graw-Hill 2012, 2293-2308.

3. Vidya Acharya N. Stem cell therapy for kidney diseasePresent and future. J Association of Physicians of India 2012;60:32-33.

4. Beaman M, Turney JH, Rodger RSC et al. Changing pattern of acute renal failure Qj med 1987;62(237):1523.

5. Butkus DE. Persistent high mortality in acute renal failure. Are we asking the right question? Arch Intern Med 1983;143:209-212.

6. Stott RB, Cameron JS. Why the persistently high mortality in acute renal failure? Lancet 1972;2:75-79.

7. Turney JH, Marshal DH. The evolution of acute renal failure. Q J Med 1990;74:83-104.

8. Prakash J, Murthy AS, Vohra R, Rajak M, Mathur SK. Acute renal failure in the intensive care unit. $\mathrm{J}$ Assoc Physicians India 2006;54:784-788.

9. Ostermann Marlies, Chang Rene WS. Acute kidney injury in the intensive care unit according to RIFLE. Critical Care Medicine 2007;35(8):1837-1843.

10. Mehta RL, Pascual MT, Soroko S et al. Spectrum of acute renal failure in the intensive care unit: The PICARD experience. Kidney International 2004;66:161321.

11. Cenzing et al. Acute Renal Failure in Central Anatolia, Nephrol Dial Transplant 2000;15:152-155.
12. Kennedy AC, Burton JA et al. Factors affecting prognosis in acute renal failure. Q J Med 1973;47:7386.

13. Kaufmann J, Dhakal M. Community acquired acute renal failure. AM J kidney disease 1991;17:191-8.

14. Metcalfe, Simpson. Acute Renal failure requiring renal replacement therapy: incidence and outcome. QJ Med 2002;95:579-583. 